\title{
GENERALIZED LIE ELEMENTS
}

\author{
RIMHAK REE
}

Introduction. Let $\lambda(i j), i, j=1,2, \ldots, m$, be $m^{2}$ elements in a field $K$ of characteristic zero such that $\lambda(i j) \lambda(j i)=1$ for all $i$ and $j$, and $x_{1}, x_{2}, \ldots, x_{m}$ non-commutative associative indeterminates over $K$. Define the elements $\left[x_{i_{1}} x_{i_{2}} \ldots x_{i_{n}}\right]$ inductively by $\left[x_{i}\right]=x_{i}$ and

$$
\left[x_{i_{1}} x_{i_{2}} \ldots x_{i_{n}}\right]=x_{i_{1}}\left[x_{i_{2}} \ldots x_{i_{n}}\right]-\prod_{\nu=2}^{n} \lambda\left(i_{1} i_{v}\right)\left[x_{i_{2}} \ldots x_{i_{n}}\right] x_{i_{1}} .
$$

Any linear combination of the elements

$$
\left[x_{i_{1}} x_{i_{2}} \ldots x_{i_{n}}\right]
$$

with coefficients in $K$ will be called a generalized Lie elememt. Generalized Lie elements reduce to ordinary Lie elements if $\lambda(i j)=1$ for all $i$ and $j$.

The purpose of this paper is to generalize to the generalized Lie elements the following: a theorem of Friedrichs, a theorem of Dynkin-Specht-Wever (2), and the Witt formula on the dimension of the space spanned by homogeneous Lie elements of a fixed degree. The set of all generalized Lie elements will be made into an algebra which generalizes the ordinary free Lie algebra. This algebra turns out to be free in a certain sense. We shall also generalize the algebra associated with shuffles in (2). ${ }^{1}$

1. Generalized Lie algebras. Throughout this paper $K$ will denote a field of characteristic zero. By a bi-character in $K$ of an additively written abelian semi-group $M$ we shall mean a map $\chi: M \times M \rightarrow K$ satisfying the following:

$$
\chi(\rho, \sigma+\tau)=\chi(\rho, \sigma) \chi(\rho, \tau), \chi(\rho+\sigma, \tau)=\chi(\rho, \tau) \chi(\sigma, \tau)
$$

for all $\rho, \sigma, \tau$ in $M$. A bi-character $\chi$ will be called skew-symmetric if $\chi(\sigma, \tau)$ $\chi(\tau, \sigma)=1$ for all $\sigma, \tau$ in $M$. An (associative or non-associative) algebra $A$ over $K$ is said to be graded by the semi-group $M$ if $A$ is a direct sum of subspaces $A_{\rho}$ indexed by $\rho \in M$ such that $f \in A_{\rho}$ and $g \in A_{\sigma}$ imply $f g \in A_{\rho+\sigma}$.

Let $L$ be an algebra graded by $M$, and let $\chi$ be a skew-symmetric bicharacter of $M$ in $K$. We shall call $L$ a generalized Lie algebra of type $\chi$, or simply a $\chi$-algebra, if $f \in L_{\rho}, g \in L_{\sigma}$, imply

$$
[f, g]+\chi(\rho, \sigma)[g, f]=0
$$

Received March 30, 1959.

'The referee remarks that the algebras considered in this paper include, as a special case, the "left Lie algebras" which are used in homological algebra (cf. for example, the exposition by P. Cartier in Séminaire Bourbaki, May, 1955). 


$$
[f,[g, h]]-\chi(\rho, \sigma)[g,[f, h]]=[[f, g], h],
$$

where $[f, g]$ denotes the product in $L$ of $f$ and $g$. In case $\chi$ is trivial, a $\chi$-algebra is clearly an ordinary Lie algebra. Let $A$ be an associative algebra graded by $M$. Define a new multiplication $[a, b]$ in the vector space $A$ by

$$
[a, b]=a b-\chi(\rho, \sigma) b a,
$$

where $a \in A_{\rho}, b \in A_{\sigma}$. Then we obtain a new algebra which we shall denote by $[A]$. It can be seen easily that $[A]$ is a $\chi$-algebra.

Let $L$ and $L^{\prime}$ be two algebras graded by the same $M$. A linear map $\phi: L \rightarrow L^{\prime}$ will be said to respect grade if $f \in L_{\rho}$ implies $\phi(f) \in L_{\rho}{ }^{\prime}$. Let $L$ be a $\chi$-algebra and $A$ an associated algebra both graded by $M$. A grade-respecting linear map $\phi: L \rightarrow A$ will be called a linearization of $L$ in $A$ if $\phi$ is a homomorphism of $L$ into $[A]$, that is, if

$$
\phi([f, g])=\phi(f) \phi(g)-\chi(\rho, \sigma) \phi(g) \phi(f)
$$

for all $f \in L_{\rho}, g \in L_{\sigma}$. The tensor algebra $T$ over the vector space $L$ is graded by $M$ if $T_{\rho}$ is defined to be the subspace spanned by elements of the form $f_{1} \otimes f_{2} \otimes \ldots \otimes f_{n}$, where $f_{i} \in L_{\rho i}$ and $\rho_{1}+\rho_{2}+\ldots+\rho_{n}=\rho$. Let $J$ be the two-sided ideal of $T$ generated by homogeneous elements of the form $f \otimes g-\chi(\rho, \sigma) g \otimes f-[f, g]$, where $f \in L_{\rho} . g \in L_{\sigma}$. Then the algebra $U=T / J$ is also graded by $M$, and the inclusion map $L \rightarrow T$ induces a linearization $\eta$ of $L$ in $U$. The algebra $U$ will be called the universal enveloping algebra of $L$; it can be characterized by the property: for any linearization $\phi: L \rightarrow A$ of $L$ into an associative algebra $A$, there exists a grade-respecting homomorphism $\xi: U \rightarrow A$ such that $\phi=\xi \circ \eta$.

2. Finitely generated free $\chi$-algebras. From now on we shall consider $\chi$-algebras $L$ satisfying the following conditions $(2.1)-(2.4)$ :

(2.1) $M$ is a free abelian group of rank $m$, with basis elements $\rho_{1}, \rho_{2}, \ldots, \rho_{m}$;

(2.2) $L_{\rho}=0$ unless $\rho$ is of the form $\rho=t_{1} \rho_{1}+t_{2} \rho_{2}+\ldots+t_{m} \rho_{m}$, where $t_{1}, t_{2}, \ldots, t_{m}$ are non-negative integers not all of which are zero;

(2.3) each $L_{\rho_{i}}(i=1,2, \ldots, m)$ is of dimension 1 ;

(2.4) $L$ is generated by $L_{\rho_{1}}, L_{\rho_{2}}, \ldots, L_{\rho_{m}}$.

A $\chi$-algebra $L$ satisfying $(2.1)-(2.4)$ above, will be called a free $\chi$-algebra of rank $m$ if any $\chi$-algebra satisfying (2.1) - (2.4) is a (grade-respecting) homomorphic image of $L$. The existence of a free $\chi$-algebra can be seen as follows: let $F$ be the free (non-associative) algebra generated by an $m$-dimensional vector space $E$ over the field $K$. If we choose a basis of $E$ over $K$, then $F$ can be graded in an obvious way by the free abelian group $M$ of rank $m$. Let $J$ be the two-sided ideal of $F$ generated by homogeneous elements of the forms $f g+\chi(\rho, \sigma) g f$ and $f(g h)-\chi(\rho, \sigma) g(f h)-(f g) h$, where $f \in F_{\rho}, g \in F_{\sigma}$. Then $L=F / J$ is easily seen to be a free $\chi$-algebra of rank $m$. 
Let $U$ be the universal enveloping algebra of the free $\chi$-algebra $L$ of rank $m$ with the linearization map $\eta: L \rightarrow U$, and let $A$ be the free associative algebra over $K$ generated by $m$ free generators $x_{1}, x_{2}, \ldots, x_{m}$. Since $L$ is free, there exists a homomorphism $\phi: L \rightarrow[A]$ such that $\phi\left(f_{i}\right)=x_{i}, i=1$, $2, \ldots, m$, that is, $\phi$ is a linearization of $L$ in $A$. Then by the definition of $U$, there exists a grade-respecting homomorphism $\xi: U \rightarrow A$ such that $\phi=\xi \circ \eta$. Then $\xi$ must be an isomorphism, since $A$ is free-associative. Thus we may regard $U$ as a free associative algebra with free generators $x_{1}=\eta\left(f_{1}\right), \ldots, x_{m}=\eta\left(f_{m}\right)$. The fact that $\eta(f)=0$ implies $F=0$ can also be proved in exactly the same way as in the case of free Lie algebras $(3,1-9)$. Hence we may identify $L$ as the subalgebra of $[U]$ generated by $x_{1}, \ldots, x_{m}$. It can be seen easily that $L$ is spanned by the elements

$$
\left[x_{i_{1}} x_{i_{2}} \ldots x_{i_{n}}\right]=\left[x_{i_{1}}\left[\ldots\left[x_{i_{n-1}} x_{i_{n}}\right] \ldots\right]\right]
$$

defined in the Introduction by using $\lambda(i j)=\chi\left(\rho_{i}, \rho_{j}\right)$. Thus we may state

Theorem 2.5. Let $K$ be a field of characteristic zero, $x_{1}, x_{2}, \ldots, x_{m}$ non-commutative associative indeterminates over $K$, and $\lambda(i j), i, j=1,2, \ldots, m$, be $m^{2}$ elements in $K$ such that $\lambda(i j) \lambda(j i)=1$ for all $i$ and $j$. Then the vector space over $K$ spanned by the elements

$$
\left[x_{i_{1}} x_{i_{2}} \ldots x_{i_{n}}\right]
$$

defined above forms a free $\chi$-algebra $L$ with respect to the multiplication

$$
\begin{aligned}
& {\left[\left[x_{i_{1}} \ldots x_{i_{p}}\right],\left[x_{j_{1}} \ldots x_{j_{q}}\right]\right]} \\
& \quad=\left[x_{i_{1}} \ldots x_{i_{p}}\right]\left[x_{j_{1}} \ldots x_{j_{q}}\right]-\prod_{\mu=1}^{p} \prod_{i=1}^{q} \lambda\left(i_{\mu} j_{\nu}\right)\left[x_{j_{1}} \ldots x_{j_{q}}\right]\left[x_{i_{1}} \ldots x_{i_{p}}\right] .
\end{aligned}
$$

The universal enveloping algebra of $L$ is isomorphic to the free associative algebra with $m$ free generators.

It should be understood in the above theorem that $L$ is graded by $M$ as follows: for $\rho=t_{1} \rho_{1}+t_{2} \rho_{2}+\ldots+t_{m} \rho_{m}, L_{\rho}$ consists of linear combinations of elements of the form

$$
\left[x_{i_{1}} x_{i_{2}} \ldots x_{i_{n}}\right]
$$

in which, for each $i, x_{i}$ appears $t_{i}$ times. Also, $\chi$ is defined by $\chi\left(\rho_{i}, \rho_{j}\right)=\lambda(i j)$.

3. A generalization of a Witt formula. Let $L$ be as in Theorem 2.5. An element in $L$ will be called a homogeneous element of degree $n$ if it is a linear combination of elements of the form

$$
\left[x_{i_{1}} x_{i_{2}} \ldots x_{i_{n}}\right] .
$$

In this section we shall compute the dimension of the space spanned by all homogeneous elements of degree $n$, following a method given by Witt (4). By the same method one may be able to compute the dimension of each $L_{\rho}$. 
Let $A$ and $B$ be two associative algebras both graded by $M$, and $\mathrm{A} \otimes B$ the tensor product of $A$ and $B$ regarded as vector spaces over $K$. Using a bi-character $\chi$ of $M$, define a multiplication in the vector space $A \otimes B$ by

$$
(a \otimes b)\left(a^{\prime} \otimes b^{\prime}\right)=\chi\left(\sigma, \rho^{\prime}\right)\left(a a^{\prime} \otimes b b^{\prime}\right)
$$

where $b \in B_{\sigma}, a^{\prime} \in A_{\rho}^{\prime}$. The algebra obtained in this way is easily seen to be associative, and will be denoted simply by $A \otimes B$. It will be used in the proof of (3.1), below, as well as in the formulation of a generalization of a theorem of Friedrichs.

Now, for the skew-symmetric bi-character $\chi$ of $M$, we have $\chi(\rho, \rho)= \pm 1$ for any $\rho \in M$. The subspace $L_{\rho}$ of the free $\chi$-algebra $L$ will be called positive or negative according as $\chi(\rho, \rho)=1$ or $\chi(\rho, \rho)=-1$. Choose a basis for each positive $L_{\rho}$ and let the union of these basis elements be $P_{1}, P_{2}, P_{3} \ldots$ Also, choose a basis for each negative $L_{\rho}$ and let the union of these basis elements be $Q_{1}, Q_{2}, Q_{3} \ldots$ Let $\eta: L \rightarrow U$ be the linearization of $L$ into its universal enveloping algebra $U$. Then we have

TheOrem 3.1. The elements

$$
\eta\left(P_{1}\right)^{s_{1}} \eta\left(P_{2}\right)^{s_{2}} \ldots \eta\left(P_{k}\right)^{s_{k}} \eta\left(Q_{1}\right)^{t_{1}} \eta\left(Q_{2}\right)^{t_{2}} \ldots \eta\left(Q_{n}\right)^{t_{n}}
$$

form a basis of the universal enveloping algebra $U$ of the free $\chi$-algebra L. Here the indices run as follows: $s_{1}, s_{2} \ldots$ are non-negative integers; each of $t_{i}$ is either 0 or $1 ; k, n=0,1,2 \ldots$

Proof. Since, for each $i$,

$$
\eta\left(\left[Q_{i}, Q_{i}\right]\right)=\eta\left(Q_{i}\right)^{2}-\chi(\rho, \rho) \eta\left(Q_{i}\right)^{2}=2 \eta\left(Q_{i}\right)^{2},
$$

it follows that $\eta\left(Q_{i}\right)^{2}$ is a linear combination of some $\eta\left(P_{j}\right)$ 's and some $\eta\left(Q_{k}\right)$ 's. Then by the definition of the linearization, it is clear that $U$ is spanned by the given elements. Thus it remains to show that the given elements are linearly independent. For this purpose, let $U^{\prime}$ be a replica of $U$ with grade-respecting isomorphism $\iota: U \rightarrow U^{\prime}$, and let $\eta^{\prime}=\iota \circ \eta$. Let $U \otimes U^{\prime}$ be the tensor product of $U$ and $U^{\prime}$ with respect to $\chi$. Then $U \otimes U^{\prime}$ is also graded by $M$ in an obvious way, and the map $\bar{\eta}: L \rightarrow U \otimes U^{\prime}$ defined by

$$
\bar{\eta}(f)=\eta(f) \otimes 1+1 \otimes \eta^{\prime}(f)
$$

is easily seen to be a linearization of $L$ into $U \otimes U^{\prime}$. Therefore there exists a homomorphism $\xi: U \rightarrow U \otimes U^{\prime}$ such that $\xi \circ \eta=\bar{\eta}$. Using $\xi$, one may now prove the linear independence of the given elements in exactly the same way as in the case of ordinary Lie algebras (3, pp. 1-8). We omit the details.

Now, let the free $\chi$-algebra $L$ given in (2.5) be graded by $M$ as in the remark following (2.5). Let the basis elements $\rho_{1}, \rho_{2}, \ldots, \rho_{m}$ be such that

$$
L_{\rho_{1}}, \ldots, L_{\rho_{p}}
$$

are positive while

$$
L_{\rho_{p+1}}, \ldots, L_{\rho_{p+q}} \text {. }
$$


$(p+q=m)$ are negative. Since, for $\rho=t_{1} \rho_{1}+\ldots+t_{m} \rho_{m}$,

$$
\chi(\rho, \rho)=\prod_{i, j} \chi\left(\rho_{i}, \rho_{j}\right)^{t_{i} t_{j}}=\prod_{i} \chi\left(\rho_{i}, \rho_{i}\right)^{t_{i}^{2}}=(-1)^{t},
$$

where $t=t_{p+1}+\ldots+t_{p+q}$, it follows that

$$
\left[x_{i_{1}} x_{i_{2}} \ldots x_{i_{n}}\right]
$$

belongs to a positive $L_{\rho}$ if and only if its degree with respect to $x_{p+1}, \ldots, x_{p+q}$ is even. Denote by $p_{n}$ and $q_{n}$, respectively, the numbers of $P_{i}$ 's of degree $n$ and the numbers of $Q_{i}$ 's of degree $n$, and consider the formal power series

$$
F(x, \lambda)=\prod_{d=1}^{\infty}\left(1+x^{d}+x^{2 d}+\ldots\right)^{p_{d}}\left(1+\lambda x^{d}\right)^{q_{d}}
$$

with a parameter $\lambda$. The coefficient $c_{n}(\lambda)$ of $x^{n}$ in $F(x)$ is a polynomial in $\lambda$ with integral coefficients. By $(3.1), c_{n}(1)$ is equal to the dimension of the subspace of $U$ spanned by all homogeneous elements of degree $n ; c_{n}(1)=(p+q)^{n}$. On the other hand, also by $(3.1), c_{n}(-1)=a_{n}-b_{n}$, where $a_{n}$ denotes the dimension of the subspace $A_{n}$ of $U$ spanned by all homogeneous elements which are of even degrees with respect to $x_{p+1}, \ldots, x_{p+q}$, and where $b_{n}$ denotes the dimension of the subspace $B_{n}$ of $U$ spanned by all homogeneous elements which are of odd degrees with respect to $x_{p+1}, \ldots, x_{p+q}$. Since $U$ is free associative, $A_{n}$ (resp. $B_{n}$ ) is spanned by elements

$$
x_{i_{1}} x_{i_{2}} \ldots x_{i_{n}}
$$

of even (resp. odd) degree with respect to $x_{p+1}, \ldots, x_{p+q}$. Thus

$$
\begin{aligned}
& a_{n}=C_{n, 0} p^{n}+C_{n, 2} p^{n-2} q^{2}+\ldots, \\
& b_{n}=C_{n, 1} p^{n-1} q+C_{n, 3} p^{n-3} q^{3}+\ldots,
\end{aligned}
$$

where $C_{n, \tau}$ are binomial coefficients. Hence $a_{n}-b_{n}=(p-q)^{n}$, and we have

$$
\begin{aligned}
& F(x, 1)=1+(p+q) x+(p+q)^{2} x^{2}+\ldots \\
& F(x, 1)=-1+(p-q) x+(p-q)^{2} x^{2}+\ldots
\end{aligned}
$$

Taking logarithms of both sides, and comparing the coefficients of $x^{n} / n$, we have, for $n=1,2, \ldots$,

$$
\begin{aligned}
& \sum_{d \mid n} d p_{d}-\sum_{d \mid n}(-1)^{n / d} d q_{d}=(p+q)^{n}, \\
& \sum_{d \mid n} d p_{d}-\sum_{d \mid n} d q_{d}=(p-q)^{n} .
\end{aligned}
$$

Let $k>0$ be an odd integer. Then, since

$$
\begin{aligned}
& \sum_{d \mid 2^{\alpha} k}(-1)^{2^{\alpha} k / d} d q_{d}=\sum_{d \mid 2^{\alpha-1} k} d q_{d}-\sum_{d \mid k} 2^{\alpha} d q_{2^{\alpha} d}, \\
& \sum_{d \mid 2^{\alpha} k} d p_{d}=\sum_{d \mid 2^{\alpha-1} k} d p_{d}+\sum_{d \mid k} 2^{\alpha} d p_{2^{\alpha}}
\end{aligned}
$$

we obtain, from the above, 


$$
\sum_{d ! k} 2^{\alpha} d\left(p_{2} \alpha_{d}+q_{2 \alpha_{d}}\right)=(p+q)^{2^{\alpha_{k}}}-(p-q)^{2^{\alpha-1} k} .
$$

Then by the Möbius inversion formula, we have

$$
p_{2 \alpha_{k}}+q_{2 \alpha_{k}}=\frac{1}{2^{\alpha} k} \sum_{d \mid k} \mu(d)\left((p+q)^{2^{\alpha_{k / d}}}-(p-q)^{2^{\alpha-1} k_{k / d}}\right) .
$$

In case $\alpha=0$, the above reduces (for odd $k$ ) to

$$
p_{k}+q_{k}=\frac{1}{k} \sum_{d \backslash k} \mu(d)(p+q)^{k / d}
$$

Following Witt, we shall use the notations:

$$
\begin{aligned}
& \psi(n)=\frac{1}{n} \sum_{d \backslash n} \mu(d)(p+q)^{n / d} ; \\
& \psi^{*}(n)=p_{n}+q_{n} .
\end{aligned}
$$

Then the above can be summarized as

TheOREM 3.2. The dimension $\psi^{*}(n)$ of the vector space spanned by all elements of the form

$$
\left[x_{i_{1}} x_{i_{2}} \ldots x_{i_{n}}\right]
$$

is given, for odd $k$, by

$$
\begin{aligned}
\psi^{*}(k) & =\psi(k) ; \\
\psi^{*}\left(2^{\alpha} k\right) & =\psi\left(2^{\alpha} k\right)+\frac{1}{2^{\alpha} k} \sum_{d ! k} \mu(d)\left((p+q)^{2^{\alpha-1} k / d}-(p-q)^{2^{\alpha-1} k / d}\right),
\end{aligned}
$$

where $p$ denotes the number of indices $i$ such that $\lambda(i i)=\chi\left(\rho_{i}, \rho_{i}\right)=1$ while $q$ denotes the number of indices $j$ such that $\lambda(j j)=-1$.

It should be remarked that the function $\psi^{*}(n)$ is completely determined by the values of $\lambda(i i)$, and independent of other values of $\lambda(i j)$. The Witt formula is obtained as the case $q=0$. In case all $\lambda(i i)=-1$, we have $p=0$, and we may deduce from the above that

$$
\psi^{*}(n)=\left\{\begin{array}{l}
\psi(n) \text { for } n \equiv 0,1,3(\bmod 4) \\
\psi(n)+\psi\left(\frac{1}{2} n\right) \text { for } n \equiv 2(\bmod 4)
\end{array}\right.
$$

4. An algebra associated with shuffles. We shall generalize the algebra defined in (2) to apply to generalized Lie elements. If $r$ and $s$ are positive integers, define a shuffle of type $(r, s)$ to be a permutation $\sigma$ of the numbers $1,2, \ldots, r+s$ such that $1 \leqslant \sigma(\mu)<\sigma(\nu) \leqslant r$ or $r<\sigma(\mu)<\sigma(\nu) \leqslant r+s$ implies $\mu<\nu$. Take $m^{2}$ elements $\lambda(i j)$ in $K$ arbitrarily, and define an algebra $A$ over $K$ as follows. $A$ has the basis

$$
\{1\} \cup\left\{a\left(i_{1} \ldots i_{n}\right) \mid i_{1}, \ldots, i_{n}=1,2, \ldots, m ; n=1,2, \ldots\right\}
$$

with the multiplication table: 1 is a unity element;

$$
a\left(i_{1} \ldots i_{r}\right) a\left(i_{r+1} \ldots i_{r+s}\right)=\sum_{\sigma} \lambda(\sigma) a\left(i_{\sigma(1)} i_{\sigma(2)} \ldots i_{\sigma(r+s)}\right)
$$


where the sum ranges over all shuffles $\sigma$ of type $(r, s)$ while $\lambda(\sigma)$ denotes the product of all $\lambda\left(i_{\sigma(\mu)}, i_{\sigma(\nu)}\right)$ such that $\mu<\nu$ and $\sigma(\mu)>\sigma(\nu)$. (We set $\lambda(\sigma)=1$ if $\sigma$ is the identity permutation.)

Thus, for example,

$$
\begin{aligned}
a(i) a(j) & =a(i j)+\lambda(j i) a(j i) ; \\
a(i) a(j k) & =a(i j k)+\lambda(j i) a(j i k)+\lambda(j i) \lambda(k i) a(j k i) .
\end{aligned}
$$

ThEOREm 4.1. The algebra $A$ is associative, and if $\lambda(i j) \lambda(j i)=1$ for all $i$ and $j$, then it satisfies the generalized commutativity:

$$
a\left(j_{1} \ldots j_{s}\right) a\left(i_{1} \ldots i_{r}\right)=\prod_{\mu=1}^{r} \prod_{\nu=1}^{s} \lambda\left(i_{\mu} j_{\nu}\right) a\left(i_{1} \ldots i_{r}\right) a\left(j_{1} \ldots j_{s}\right) .
$$

Proof. If

$$
f=a\left(i_{1} \ldots i_{r}\right), g=a\left(i_{r+1} \ldots i_{r+s}\right), h=a\left(i_{r+s+1} \ldots i_{r+s+t}\right) .
$$

then it is readily seen that both $(f g) h$ and $f(g h)$ are of the form

$$
\sum \lambda(\sigma) a\left(i_{\sigma(1)} i_{\sigma(2)} \ldots i_{\sigma(r+s+t)}\right)
$$

where $\sigma$ runs over all permutations of $1,2, \ldots, r+s+t$ such that any one of the three conditions

$$
\begin{aligned}
1 \leqslant \sigma(\mu) & <\sigma(\nu)<r, \\
r<\sigma(\mu) & <\sigma(\nu) \leqslant r+s, \\
r+s<\sigma(\mu) & <\sigma(\nu)<r+s+t
\end{aligned}
$$

implies $\mu<\nu$, and where $\lambda(\sigma)$ denotes the product of all $\lambda\left(i_{\sigma(\mu)} i_{\sigma(\nu)}\right)$ such that $\mu<\nu$ and $\sigma(\mu)>\sigma(\nu)$. Hence $(f g) h=f(g h)$. The second half of the theorem may be verified easily.

In the rest of this section, we shall assume that $\lambda(i j) \lambda(j i)=1$ for all $i$ and $j$. Making the convention that $a\left(i_{1} \ldots i_{r}\right)$ stands for 1 whenever the set $\left\{i_{1}, \ldots, i_{r}\right\}$ of indices is empty, we define the bilinear operation $\vee$ in $A$ by

$$
a\left(i_{1} \ldots i_{r}\right) \vee a\left(j_{1} \ldots j_{s}\right)=a\left(i_{1} \ldots i_{r} j_{1} \ldots j_{s}\right) .
$$

We also make the convention that the multiplication in $A$ has priority over the operation $\vee$.

Define the elements $a\left[i_{1} i_{2} \ldots i_{n}\right]$ in $A$ inductively by $a[i]=a(i)$ and

$$
a\left[i_{1} i_{2} \ldots i_{n}\right]=a\left(i_{1}\right) \vee a\left[i_{2} \ldots i_{n}\right]-\prod_{\nu=1}^{n-1} \lambda\left(i_{n} i_{v}\right) a\left(i_{n}\right) \vee a\left[i_{1} \ldots i_{n-1}\right] .
$$

For the generalizations in the next section of some theorems on Lie elements, we need the following

Theorem 4.2. For $n>0$, we have

$$
\sum_{s=1}^{n} a\left[i_{1} \ldots i_{s}\right] a\left(i_{s+1} \ldots i_{n}\right)=n a\left(i_{1} \ldots i_{n}\right) \text {. }
$$


The above theorem may be proved in exactly the same way as in the case where all $\lambda(i j)=1$ (2), if we use the linear map $D: A \rightarrow A$ defined by $D(1)=0$ and

$$
D a\left(i_{1} i_{2} \ldots i_{n}\right)=\gamma\left(i_{1}\right) a\left(i_{2} \ldots i_{n}\right),
$$

where $\gamma(1), \ldots, \gamma(m)$ are $m$ arbitrary elements in $K$. We omit the proof of (4.2). Incidentally, the map $D$ becomes an anti-derivation of $A$ if all $\lambda(i j)=-1$.

Theorem 4.3. If the linear map $\phi: A \rightarrow A$ is defined by $\phi(1)=0$ and $\phi\left(a\left(i_{1} i_{2} \ldots i_{n}\right)\right)=a\left[i_{1} i_{2} \ldots i_{n}\right]$, then $\phi\left(a\left(i_{1} \ldots i_{r}\right) a\left(i_{r+1} \ldots i_{r+s}\right)\right)=0$ for all $i_{1}, i_{2}, \ldots, i_{r+s}=1,2, \ldots, m ; r>0, s>0$.

Proof. We shall proceed by induction on $n=r+s$. If $n=2$, then the theorem can be verified easily. Assume $n>2$ and that the theorem is proved for smaller values of $n$. By the definition of the multiplication in $A$, we have

$$
\begin{aligned}
& \phi\left(a\left(i_{1} \ldots i_{r}\right) a\left(i_{r+1} \ldots i_{n}\right)\right) \\
& =\sum \lambda(\sigma) a\left(i_{\sigma(1)}\right) \vee a\left[i_{\sigma(2)} \ldots i_{\sigma(n)}\right] \\
& \quad-\sum \lambda(\sigma) \prod_{\nu=1}^{n-1} \lambda\left(i_{\sigma(n)} i_{\sigma(v)}\right) a\left(i_{\sigma(n)}\right) \vee a\left[i_{\sigma(1)} \ldots i_{\sigma(n-1)}\right],
\end{aligned}
$$

where the sums run over all shuffles of type $(r, s), r+s=n$. Since $\sigma(1)=1$ or $r+1$, and $\sigma(n)=r$ or $n$, the right-hand side of the above equation can be written

$$
\begin{aligned}
\sum_{\sigma(1)=1} \lambda(\sigma) a\left(i_{1}\right) & \vee a\left[i_{\sigma(2)} \ldots i_{\sigma(n)}\right] \\
& +\sum_{\sigma(1)=r+1} \lambda(\sigma) a\left(i_{r+1}\right) \vee a\left[i_{\sigma(2)} \ldots i_{\sigma(n)}\right] \\
& -\sum_{\sigma(n)=r} \lambda(\sigma) \prod_{\nu=1}^{n-1} \lambda\left(i_{r} i_{\sigma(v)}\right) a\left(i_{r}\right) \vee a\left[i_{\sigma(1)} \ldots i_{\sigma(n-1)}\right] \\
& -\sum_{\sigma(n)=n} \lambda(\sigma) \prod_{\nu=1}^{n-1} \lambda\left(i_{n} i_{\sigma(v)}\right) a\left(i_{n}\right) \vee a\left[i_{\sigma(1)} \ldots i_{\sigma(n-1)}\right] \\
=a\left(i_{1}\right) & \vee \phi\left(a\left(i_{2} \ldots i_{r}\right) a\left(i_{r+1} \ldots i_{n}\right)\right) \\
& +\prod_{\nu=1}^{r} \lambda\left(i_{r+1} i_{v}\right) a\left(i_{r+1}\right) \vee \phi\left(a\left(i_{1} \ldots i_{r}\right) a\left(i_{r+2} \ldots i_{n}\right)\right) \\
& -\prod_{\mu=r+1}^{n} \lambda\left(i_{\mu} i_{r}\right) \prod_{\nu=1, \neq>}^{n} \lambda\left(i_{r} i_{v}\right) a\left(i_{r}\right) \vee \phi\left(a\left(i_{1} \ldots i_{r-1}\right) a\left(i_{r+1} \ldots i_{n}\right)\right) \\
& -\prod_{\nu=1}^{r-1} \lambda\left(i_{n} i_{v}\right) a\left(i_{n}\right) \vee \phi\left(a\left(i_{1} \ldots i_{\tau}\right) a\left(i_{r+1} \ldots i_{n-1}\right)\right)
\end{aligned}
$$

$=0$ 
because of the induction assumption and the fact that, for $r=1$,

$$
\prod_{\mu=r+1}^{n} \lambda\left(i_{\mu} i_{r}\right) \prod_{\nu=1, \neq r}^{n} \lambda\left(i_{r} i_{\nu}\right)=1
$$

Corollary 4.3. If $0<r<n$, then

$$
n a\left(i_{1}, \ldots, i_{r}\right) a\left(i_{r+1} \ldots i_{n}\right)=\sum \lambda(\sigma)\left(n a\left(i_{\sigma(1)} \ldots i_{\sigma(n)}\right)-a\left[i_{\sigma(1)} \ldots i_{\sigma(n)}\right]\right),
$$

where the sum ranges over all shuffles of type $(r, n-r)$.

The above corollary, together with $(4.2)$, shows that the $(n-1) m^{n}$ elements $a\left(i_{1} \ldots i_{r}\right) a\left(i_{r+1} \ldots i_{n}\right), i_{1}, \ldots, i_{n}=1,2, \ldots, m ; 0<r<n$, and the $m^{n}$ elements $n a\left(i_{1} \ldots i_{n}\right)-a\left[i_{1} \ldots i_{n}\right]$ span the same vector space over $K$. Also from (4.2) we obtain

Corollary 4.4. The linear map $\phi_{0}: A \rightarrow A$ defined by $\phi_{0}(1)=0$ and

$$
\phi_{0}\left(a\left(i_{1} i_{2} \ldots i_{n}\right)\right)=n^{-1} a\left[i_{1} i_{2} \ldots i_{n}\right] \text {, }
$$

for $n>0$, is a projection, that is, $\phi_{0}{ }^{2}=\phi_{0}$.

The following theorem is essentially a generalization of Theorem 2.6 of (2), and may be proved by using the map $D$ introduced in the above.

TheOREM 4.5. For $n>0$, we have

$$
\sum_{s=0}^{n}(-1)^{s} \prod_{s<\mu<\nu<n} \lambda\left(i_{\nu} i_{\mu}\right) a\left(i_{1} \ldots i_{s}\right) a\left(i_{n} i_{n-1} \ldots i_{s+1}\right)=0 .
$$

5. Generalization of a theorem of Friedrichs. Let $L$ be a free $\chi$ algebra of rank $m$, and $\eta: L \rightarrow U$ the linearization of $L$ into its universal enveloping algebra. Let $U^{\prime}$ be a replica of $U$ with the grade-respecting isomorphism $\iota: U \rightarrow U^{\prime}$ and $\eta^{\prime}=\iota \circ \eta$. Let $U \otimes U^{\prime}$ be the tensor product of $U$ and $U^{\prime}$ with respect to $\chi$. In the course of the proof of (3.1) we have seen that the map $\bar{\eta}: L \rightarrow U \otimes U^{\prime}$ defined by

$$
\bar{\eta}(f)=\eta(f) \otimes 1+1 \otimes \eta^{\prime}(f)
$$

is a linearization and that there exists a homomorphism $\xi: U \rightarrow U \otimes U^{\prime}$ such that $\xi \circ \eta=\bar{\eta}$. Now the following theorem generalizes a theorem of Friedrichs (2).

Theorem 5.1. Let $\eta, \iota$, and $\xi$ be as above. Then an element $u$ in $U$ belongs to the image $\eta(L)$ of $L$ under $\eta$ if and only if

$$
\xi(u)=u \otimes 1+1 \otimes \iota(u) .
$$

Proof. The "only if" part follows from the fact that $\bar{\eta}=\xi \circ \eta$. In order to prove the "if" part, let $x_{1}, x_{2}, \ldots, x_{m}$ be free generators of $U$ and write, for simplicity, $x_{i}$ and $x_{i}{ }^{\prime}$ for $x_{i} \otimes 1$ and $1 \otimes \iota\left(x_{i}\right)$, respectively. If

$$
u=\sum \alpha_{i_{1} \ldots i_{n}} x_{i_{1}} \ldots x_{i_{n}}
$$


with coefficients in $K$, then

$$
\begin{aligned}
\xi(u) & =\sum \alpha_{i_{1} \ldots i_{n}}\left(x_{i_{1}}+x_{i_{1}}^{\prime}\right) \ldots\left(x_{i_{n}}+x_{i_{n}}^{\prime}\right) \\
& =\sum \sum_{s=0}^{n} \phi\left(a\left(i_{1} \ldots i_{s}\right) a\left(i_{s+1} \ldots i_{n}\right)\right) x_{i_{1}} \ldots x_{i_{s}} x_{i_{s+1}}^{\prime} \ldots x_{i_{n}}^{\prime},
\end{aligned}
$$

where $\phi$ is a linear map: $A_{n} \rightarrow K$ defined by

$$
\phi\left(a\left(i_{1} \ldots i_{n}\right)\right)=\alpha_{i_{1}} \ldots i_{n} .
$$

Hence the condition given in (5.1) is equivalent to

$$
\phi\left(a\left(i_{1} \ldots i_{s}\right) a\left(i_{s+1} \ldots i_{n}\right)\right)=0 \quad(0<s<n) .
$$

The rest of the proof is exactly the same as in the case $\lambda(i j)=1$ (2, p. 214), and may be omitted. Here we have to use

$$
\sum a\left[i_{1} \ldots i_{n}\right] x_{i_{1}} \ldots x_{i_{n}}=\sum a\left(i_{1} \ldots i_{n}\right)\left[x_{i_{1}} \ldots x_{i_{n}}\right]
$$

but this, too, can be proved as in (2, p. 213).

Similarly we may prove the following

Theorem 5.2. A homogeneous element

$$
f=\sum \alpha_{i_{1} \ldots i_{n}} x_{i_{1}} \ldots x_{i_{n}}
$$

in $C$ of degree $n>0$ is a generalized Lie element if and only if

$$
n f=\sum \alpha_{i_{1} \ldots i_{n}}\left[x_{i_{1}} \ldots x_{i_{n}}\right] .
$$

This generalizes a theorem of Dynkin-Specht-Wever (2, p. 214).

\section{REFERENCES}

1. C. Chevalley, Fundamental concepts of algebra (New York: Academic Press Inc., 1956).

2. Rimhak Ree, Lie elements and an algebra associated with shuffles, Ann. Math., 68 (1958), $210-220$.

3. Seminaire "Sophus Lie," Theorie des algebres de Lie et topologie des groupes de Lie, 1954-5.

4. Ernst Witt, Treue Darstellung Liescher Ringe, J. Reine Angew. Math., 177 (1937), 152-160.

University of British Columbia 